\title{
OBSERVACIONES SOBRE LOS DINOFLAGELADOS (Pyrrhophyta) de las Costas de Castellón (ESPAÑA).
}

\author{
Simón Juan ROVIRA FORCADA
}

\begin{abstract}
RESUMEN: En este trabajo se presentan 27 especies de dinoflagelados (Pyrrhophyta), procedentes de muestreos realizados durante el otoño de 1989, en la zona nerítica del Mediterráneo frente al puerto de Castellón (U.T.M. 31S BE451293). Se comentan algunas características morfométricas de especies pertenecientes a los géneros: Prorocentrum, Amphisolenia, Dinophisis, Ornithocercus, Peridinium, Ceratocorys y Ceratium. La especie Dinophisis schuetti Murray et Whitting, 1899, se cita por primera vez para esta costa.
\end{abstract}

Palabras clave: Dinoflagelados, Fitoplancton, Mediterráneo, zona nerítica, Pyrrhophyta.

ABSTRACT: In the present work, 27 species of dinoflagellates (Pyrrhophyta) were determinated. The samples were taken in the 1989 autumn in the Mediterranean neritic zone near the harbour of Castellón (Eastern coast of Spain) (U.T.M. 31S BE451293). Morphometrical characteres of the species are coment. The species are incluse in the genus: Prorocentrum, Amphisolenia, Dinophysis, Ornithocercus, Peridinium, Ceratocorys and Ceratium. The specie Dinophisis schuetti Murray et Whitting, 1899 is reported for the first time in this coast.

Key words: Dinoflagellates, Mediterranean Sea, Neritic zone, phytoplankton, Pyrrhophyta.

\section{INTRODUCCIÓN}

Las costas de Castellón ya fueron estudiadas entre 1955 y 1975 por los autores Herrera \& al. (1955, 57, 61, 63), Margalef \& al. (1957, 59, 63, 64), Muñoz \& al. (1956, 72) y San Feliú \& al. (1967, 70, 75).

En estos trabajos se procedió a la identificación e inventariado de las especies fitoplanctónicas, siendo encontrados más de cien dinoflagelados. Se estudió también la riqueza de la zona mediante la productividad, relacionándola con factores ambientales (tales como la temperatura del agua en superficie, dirección y velocidad del viento, 
nivel del mar, etc) y fenómenos de afloramientos o movimientos de masas de agua que provocarían crecimientos y cambios en la composición relativa del fitoplancton.

El objetivo fundamental del presente estudio ha sido caracterizar y determinar las especies de dinoflagelados existentes en la zona nerítica de las costas castellonenses, trabajo que esperamos ampliar en futuras campañas.

\section{MATERIAL Y MÉTODO}

El área de muestreo se restringió a la zona nerítica frente al puerto de Castellón (situación U.T.M. 31S BE451293), a una distancia aproximada de 5 millas de la costa (fig. 1).

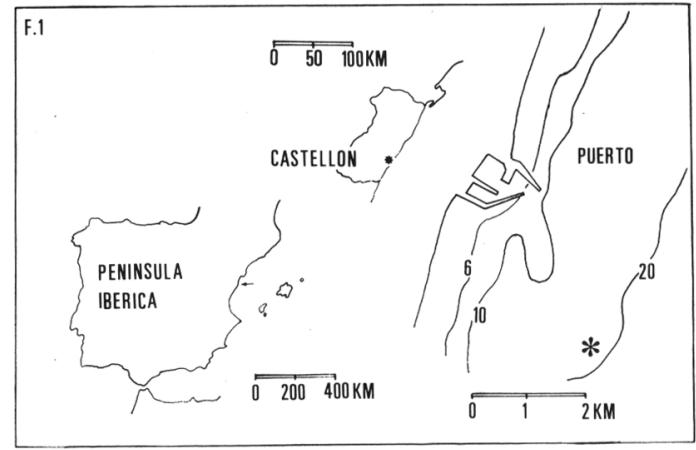

Figura 1: Croquis que muestra la situación del Puerto de Castellón y el área de muestreo (asterisco).

Las muestras fueron tomadas durante los meses de Octubre y Noviembre de 1989. Durante estos meses ya comienza el enfriamiento de las aguas superficiales (Margalef \& Herrera, 1963) que conducirá a los meses fríos, con mayor abundancia de fitoplancton gracias al ascenso de aguas ricas en nutrientes (Margalef \& al., 1957).

Los muestreos consistieron en diversos arrastres, siempre antes del mediodía, a una profundidad entre 0.5 y 1.5 metros.

La red utilizada para el muestreo es del tipo troncocónica, con una luz de poro de $80 \mu \mathrm{m}$, contruida según las indicaciones del «Phytoplankton manual» (Unesco, 1981).

El material recolectado era fijado inmediatamente añadiendo formaldehído neutralizado con hexametilenotetramina.

La determinación fue realizada con la ayuda de un microscopio óptico Olympus IM, provisto de ocular micromético y equipo fotográfico, empleándose las claves clásicas de Rabenhorst (1933-1937) y Tregouboff (1957) y las más modernas de Dodge (1985) y Rampi \& Bernhard (1980), habiendo consultado los trabajos de López (1955 y 1966) para el género Ceratium. 


\section{RESULTADOS}

Los táxones han sido ordenados siguiendo la clasificación sugerida por Spector (1984) (en Dodge, 1985).

\section{ORDEN PROROCENTRALES}

Familia Prorocentraceae

- Género Prorocentrum Ehrenberg, 1833.

* Prorocentrum micans Ehrenberg, 1833.

(Sin.: Cercaria Michaelis, 1833. P. viride Ehrenberg, 1840).

Se trata de un dinoflagelado muy común en áreas portuarias y zona nerítica en general (Dodge, 1985). Ya lo citan Herrera \& Margalef (1955) y es común en trabajos posteriores durante los meses de Octubre y Noviembre. Margalef \& al. (1955) lo hallaron abundante en el Puerto de Vigo en Octubre de 1954. Fue hallado en los dos meses de muestreo.

Dimensiones: $45 \mu \mathrm{m}$ x $35 \mu \mathrm{m}$, con un cuerno de $9 \mu \mathrm{m}$. Lám. 1, a.

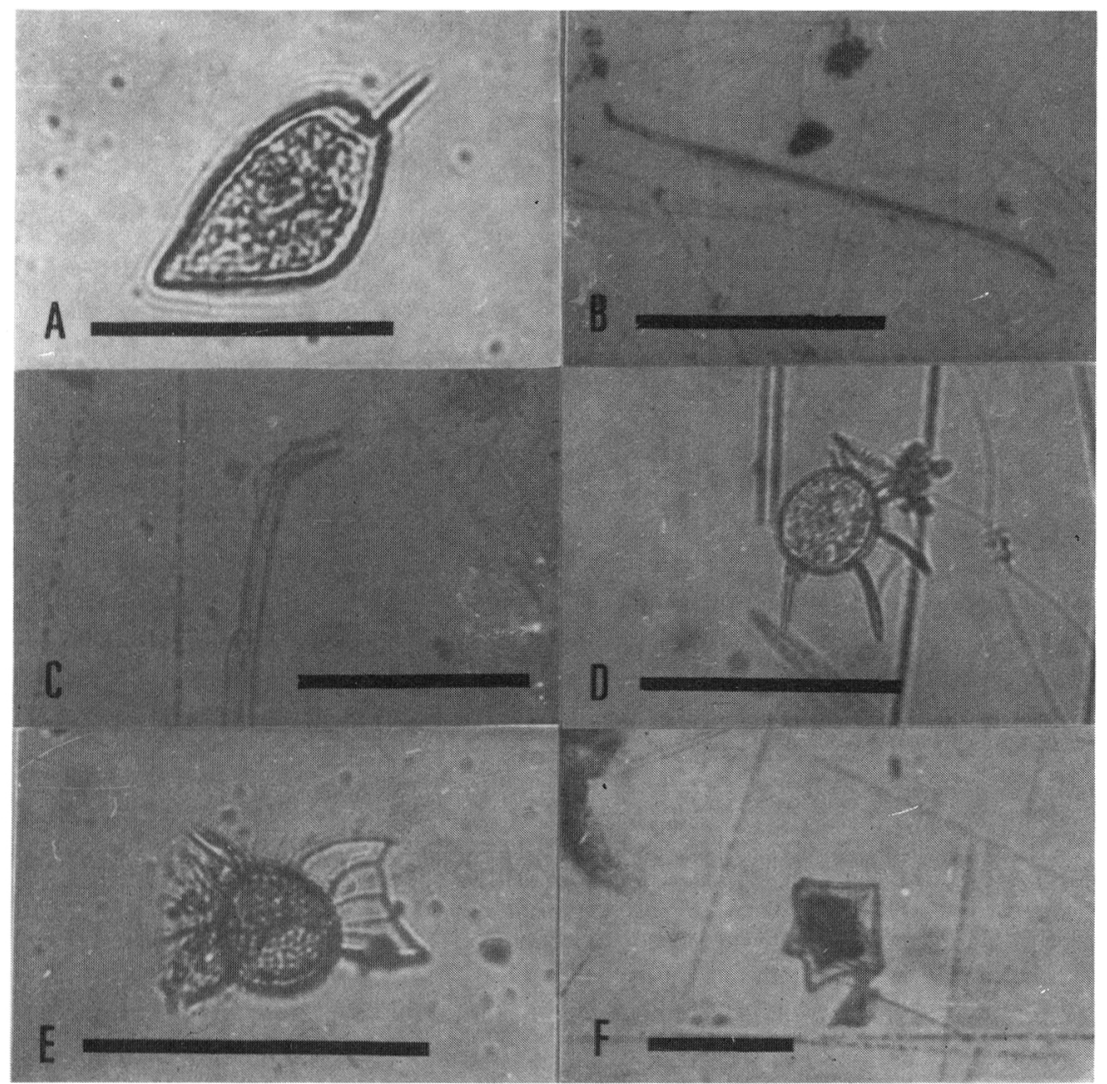

Lámina 1: (Tras en nombre se indica el tamaño de la barra); a) Prorocentrum micans. $500 \mu \mathrm{m}$; b) Amphisolenia bidentata. $500 \mu \mathrm{m}$; c) Amphisolenia bidentata. Detalle. $100 \mu \mathrm{m}$; d) Dinophisis schuetti. $100 \mu \mathrm{m}$; e) Ornithocercus heteroporus. $100 \mu \mathrm{m}$; f) Peridium conicum. $100 \mu \mathrm{m}$. 


\section{ORDEN DINOPHISIALES}

Familia Amphisoleniaceae

- Género Amphisolenia Stein, 1983.

* Amphisolenia bidentata Schröter, 1900.

(Sin.: Amphisolenia sp. Shun, 1903).

Encontrada en Octubre de 1957 (Margalef \& al., 1959). Presenta gran longitud, con la epiteca desplazada a un extremo y unos dientes en la zona antiapical de la hipoteca. Encontrado en las muestras de Noviembre.

Longitud de $1000 \mu \mathrm{m}$, anchura en la epiteca de $40 \mu \mathrm{m}$. Lám. 1, b y c.

Familia Dinophisiaceae

- Género Dinophisis Ehrenberg, 1839.

* Dinophisis schuetti Murray et Whitting, 1899.

(Sin.: Dinophisis uracantha Schütt, 1895. D. schuetti var. uracanthoides Forti et Issel, 1924).

Esta especie no ha sido nombrada como tal en Castellón en ninguno de los trabajos revisados para el presente artículo, si bien Margalef \& al. (1957) identifican a Dinophisis hastata Stein ( $=$ D. uracantha var. mediterranea Joergens) (dimensiones: $4 \mu \mathrm{m}$ epiteca, $42 \mu \mathrm{m}$ hipoteca, $14 \mu \mathrm{m}$ costilla antiapical). Aunque existe una semejanza, creemos que son especies diferentes y puede darse como primera observación para Castellón de Dinophisis schuetti (ver fig. 2). Se encontró en las muestras de Noviembre.

Dimensiones: $15 \mu \mathrm{m}$ epiteca, $40 \mu \mathrm{m}$ hipoteca, $25 \mu \mathrm{m}$ costilla antiapical. Lám. 1, d.

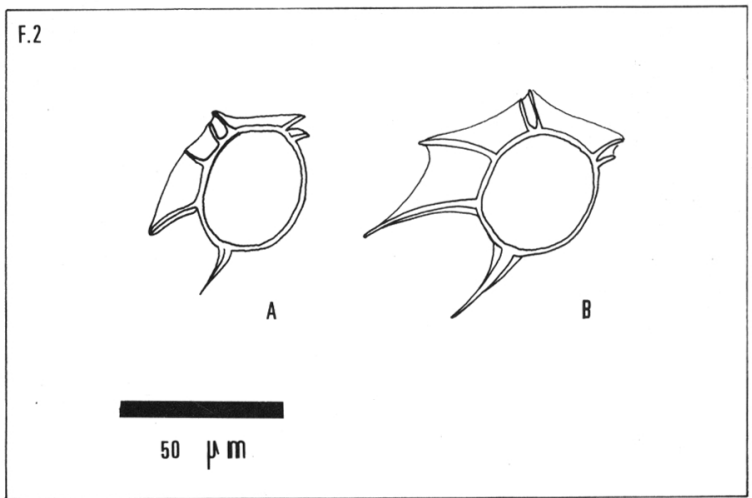

Figura 2: Comparación entre Dinophisis hastata Stein, identificado por Margalef \& al. (1957) (A) y Dinophisis schuetti Murray et Whitting identificado en el presente trabajo (B). D. hastata se ha redibujado a partir de una ilustración del trabajo citado, el dibujo de $D$. schuetti es original.

Familia Ornithocercaceae

Género Ornithocercus Stein, 1883.

* Ornithocercus heteroporus Kofoid, 1907.

Pese a su parecido con O. magnificus Stein, 1883, el O. heteroporus pertenece al grupo Splendidus y el O. magnificus al grupo Magnificus (Rabenhosrt, 1933). Margalef \& al. (1959) lo encontraron en Castellón en Noviembre de 1957. Hallado en las muestras de Noviembre.

Dimensiones: $12 \mu \mathrm{m}$ epit., $40 \mu \mathrm{m}$ hipot., $25 \mu \mathrm{m}$ costilla antiapical. Lám. 1, e. 


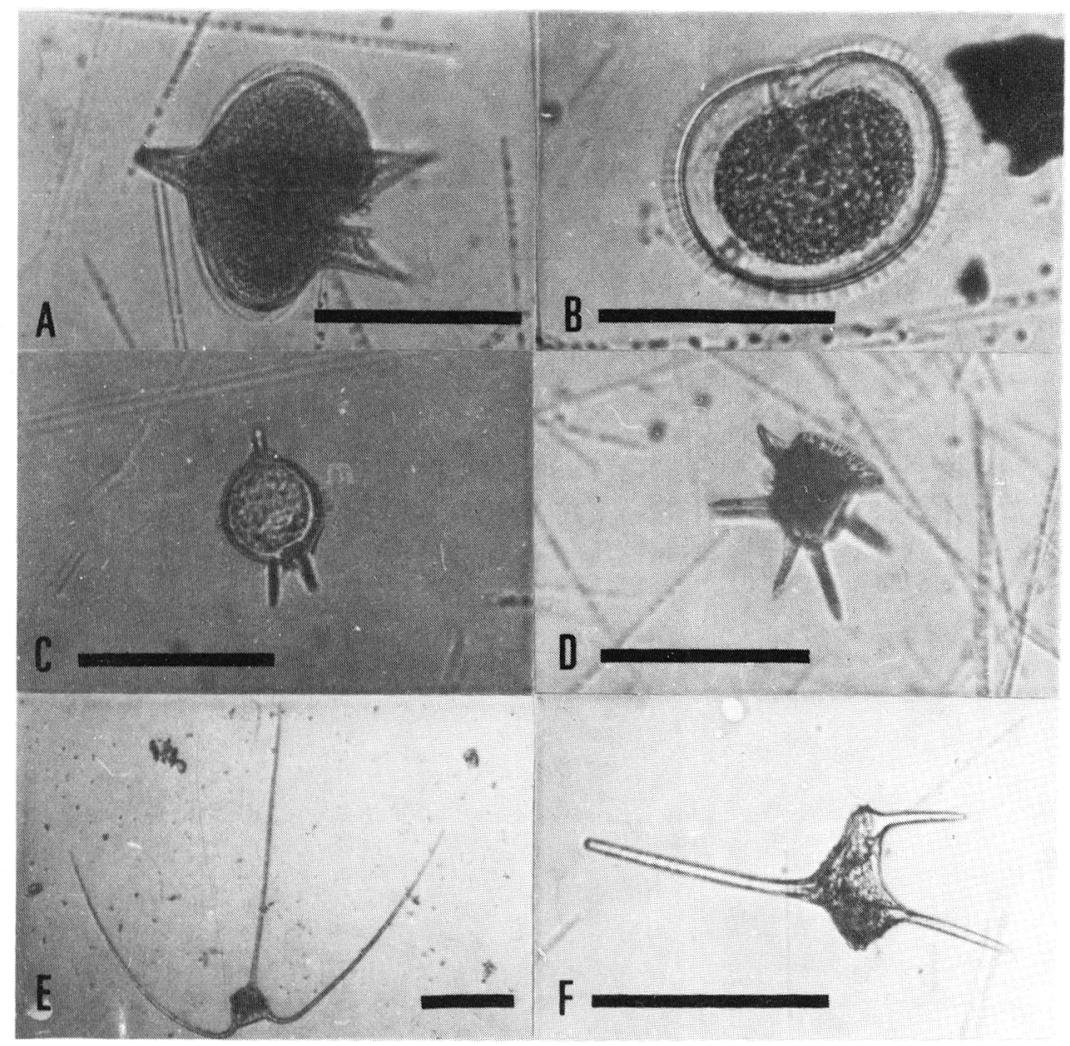

Lámina 2: a) Peridinium depressum. $100 \mu \mathrm{m}$; b) Peridinium depressum. Vista apical. $100 \mu \mathrm{m}$; c) Peridinium steinii. $100 \mu \mathrm{m}$; d) Ceratocorys horrida. $100 \mu \mathrm{m}$; e) Ceratium buceros. $100 \mu \mathrm{m}$; f) Ceratium candelabrum var depressum. $100 \mu \mathrm{m}$.

\section{ORDEN PERIDINIALES}

\section{Familia Peridiniaceae}

- Género Peridinium Ehrenberg, 1832 (= Protoperidinium Bergh, 1881).

* Peridinium conicum (Gran) Ostenfeld et Schmidt, 1900.

(Sin.: Peridinium acutangulum (Lemm.) Jörgensen, 1913).

Encontrado por Herrera \& Margalef (1961) durante Octubre de 1959. En esta ocación ha aparecido en las muestras de Noviembre. Longitud de $85 \mu \mathrm{m}$, diámetro en el cínculo de 75 $\mu \mathrm{m}$. Lám. 1, f.

* Peridinium depressum (Bailey) Balech.

(Sin.: Peridinium parallelum Broch, 1906. P. divergens Vanhöffen, 1897. P. marinum Lindemann, 1925).

Encontrado por Muñoz \& al. (1956) en ambos meses. En nuestro caso apareció en Noviembre. 
Longitud de $135 \mu \mathrm{m}$ y diámetro en el cínculo de $130 \mu \mathrm{m}$. Lám. 2, a y 2, b.

* Peridinium steinii (Jörgensen) Balech.

(Sin.: Peridinium michaelis Stein, 1883. P. pellucidum Ramsay-Wright, 1907. P. micrapium Meunier, 1919).

Citado por vez primera en los inventarios de Muñoz \& al. (1956), durante el mes de Octubre. Fue encontrado en muestras de Noviembre.

Longitud de $85 \mu \mathrm{m}$, anchura de $50 \mu \mathrm{m}$. Lám. 2, c.

Familia Ceratoceraceae

- Género Ceratocorys Stein, 1883.

* Ceratocorys horrida Stein, 1883.

(Sin.: Ceratocorys tridentata Daday, 1888. C. spinifera Murray et Whitting, 1899. Dinophisis jourdani Gourret, 1883).

Lo citan Herrara \& Margalef (1957) durante el mes de Octubre. Apareció en las muestras de Noviembre.

Longitud total de $75 \mu \mathrm{m}$, diámetro en el cínculo de $45 \mu \mathrm{m}$, costillas de $30 \mu \mathrm{m}$. Lám. 2, d.

Familia Ceratiaceae

- Género Ceratium Schranck, 1793.

* Ceratium buceros Zach, 1906.

(Sin.: C. horridum Gran, Jörgensen, 1920. C. tenue (Ostf. et Schmidt) Jörg, 1911. C. patentissimum Karsten, 1906. C. tripos inversum Karsten, 1907. C. tripos inclinatum Kofoid, 1907. C. leptosomum Jörgensen, 1911. C. claviger Kofoid, 1907. C. undulatum Schröder, 1906).

Encontrado por Muñoz \& al. (1956) en Octubre. Aparece en las muestras de ambos meses.

Longitud de $320 \mu \mathrm{m}$, diámetro cingular de $50 \mu \mathrm{m}$, cuernos de 250 y $300 \mu \mathrm{m}$. Lám. 2, e. * Ceratium candelabrum (Ehrenberg) Stein, 1883 var. depressum (Pouchet) Jörgensen, 1920.

(Sin.: Peridinium candelabrum Ehrenberg, 1859. C. furca var. depressa Pouchet, 1883. C. globatum Gourret, 1883. C. dilatatum var. parvum Gourret, 1883).

Citado por Herrera et al. (1955) en Octubre y Noviembre. Lo encontramos durante los dos meses.

Long. de $180 \mu \mathrm{m}$, pico de $150 \mu \mathrm{m}$, cíngulo con $80 \mu \mathrm{m}$, cuernos de 60 y $75 \mu \mathrm{m}$. Lám. 2,f. * Ceratium carriense Gourret, 1883.

(Sin.: C.tripos var. massiliense Gourret, 1883.C.volans Pavillard, 1905. C. massiliense Pavillard, 1907).

Hallado por Muñoz \& al. (1956) en ambos meses. Presente en muestras de Octubre.

Long. de $530 \mu \mathrm{m}$, pico de $410 \mu \mathrm{m}$, cíngulo de $80 \mu \mathrm{m}$, cuernos de 630 y $780 \mu \mathrm{m}$. Lám. 3,a.

* Ceratium carriense Gourret, 1883 var. volans (Cleve) Sournia.

(Sin.: Ceratium volans Cleve, 1900. C. patentissimum Ostf. et Schmidt, 1901).

Encontrado por Herrera \& Margalef (1957) durante el mes de Octubre. Lo hemos encontrado durante el mismo mes.

Long. de $630 \mu \mathrm{m}$, pico de $530 \mu \mathrm{m}$, cíngulo de $80 \mu \mathrm{m}$, cuernos de 660 y $910 \mu \mathrm{m}$. Lám. 3,b.

* Ceratium concilians Jörgensen, 1920.

(Sin.: Ceratium tripos va. contortum Gourret, 1883. C. tr. gracilis Entz, 1902. C. gibberum f. sinistrum Jörgensen, 1911).

Citado por Herrera \& al. (1955) durante ambos meses. Presente en las muestras de Noviembre.

Longitud de $310 \mu \mathrm{m}$, pico de $240 \mu \mathrm{m}$, cíngulo de $70 \mu \mathrm{m}$, cuerno visible de $105 \mu \mathrm{m}$. Lám. 3, c. 


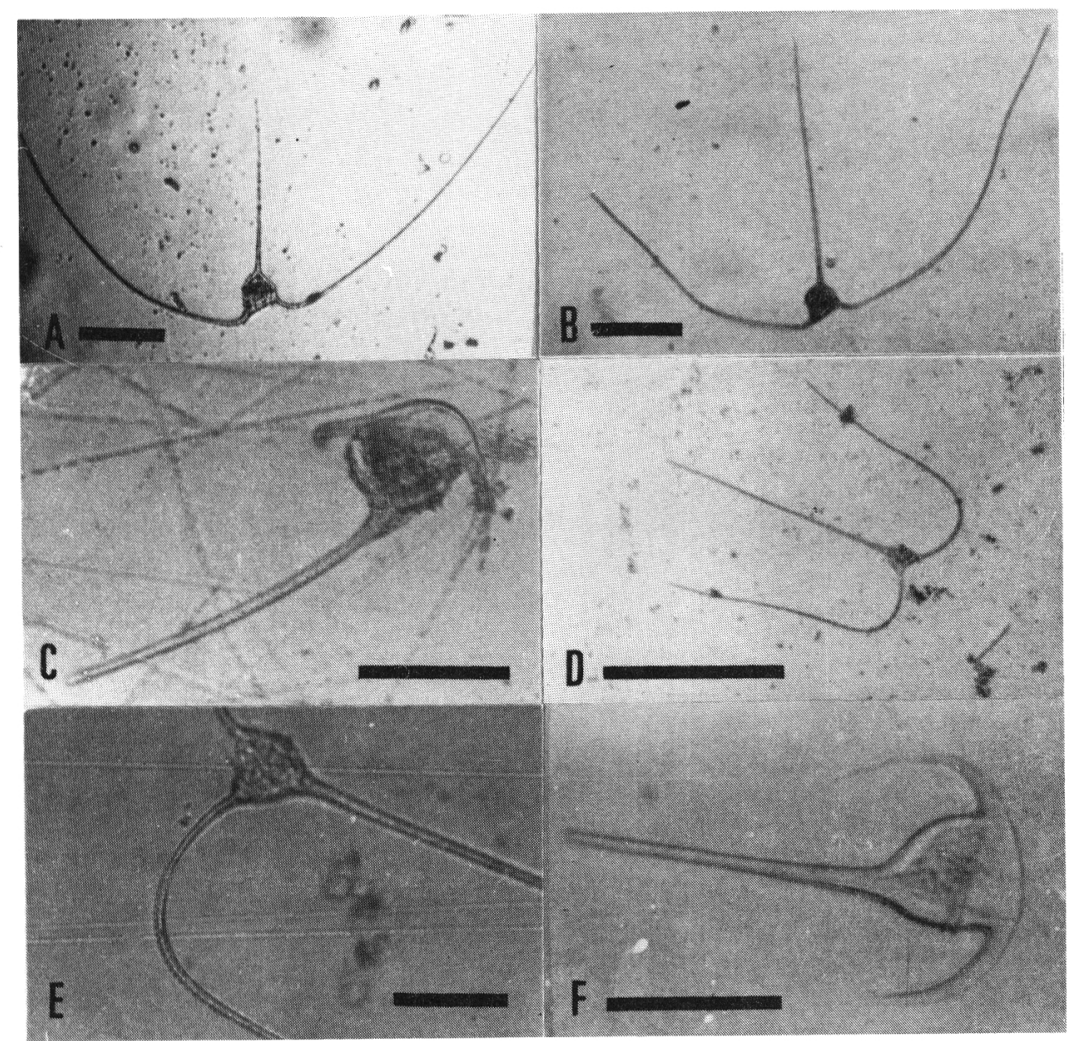

Lámina 3: a) Ceratium carriense. $200 \mu \mathrm{m}$; b) Ceratium carriense var volans. $200 \mu \mathrm{m}$; c) Ceratium concilians. $100 \mu \mathrm{m}$; d) Ceratium contrarium. $500 \mu \mathrm{m}$; e) Ceratium contrarium. Detalle. 100 $\mu \mathrm{m}$; f) Ceratium declinatum. $100 \mu \mathrm{m}$.

* Ceratium contrarium (Gourret) Pavillard, 1905.

(Sin.: Ceratium trichoceros var. contrarium (Gourr.) Schiller. C. inflexum (Gourr.) Kofoid, Jörgensen, 1911. C. flagelliferum Cleve, 1901).

Encontrado durante ambos meses por Herreta \& al. (1955). Apareció en muestras de Octubre.

Longitud de $715 \mu \mathrm{m}$, pico de $630 \mu \mathrm{m}$, cíngulo de $65 \mu \mathrm{m}$, cuernos de $780 \mu \mathrm{m}$. Lám. 3 , d y 3 , e.

* Ceratium declinatum (Karsten) Jörgensen, 1911.

(Sin.: Ceratium tripos var. gracile Schrödder, 1900. C.tripos Pavillard, 1905. C. gracile Pavillard, 1905. C. tripos heterocamptum Karsten, 1907).

Citado por Herrera \& al. (1955) durante ambos meses. Presente en nuestras muestras de Octubre.

Long. de $250 \mu \mathrm{m}$, pico de $165 \mu \mathrm{m}$, cíngulo de $50 \mu \mathrm{m}$, cuernos de 45 y $55 \mu \mathrm{m}$. Lám. 3, f. 


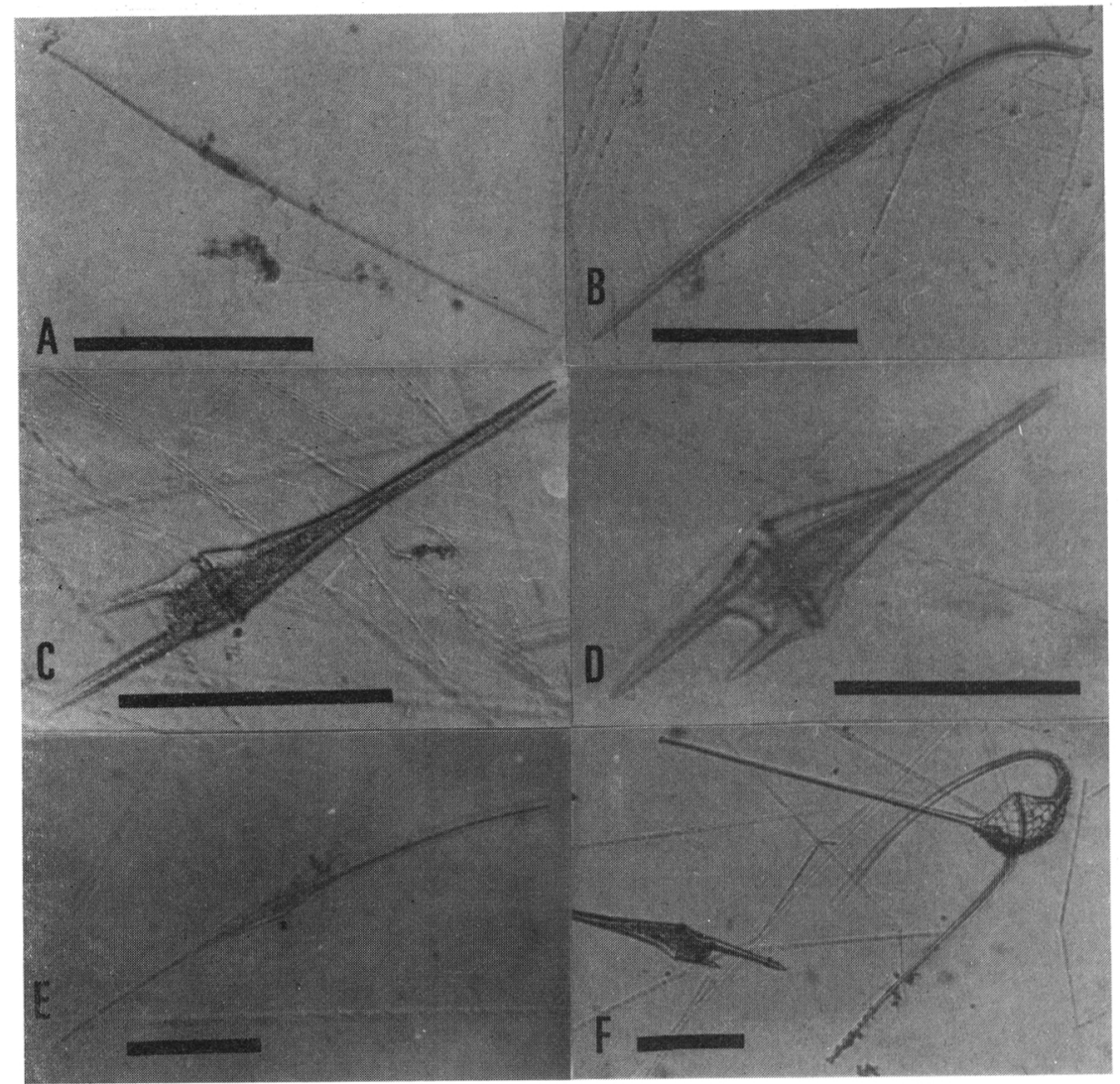

Lámina 4: a) Ceratium extensum. $500 \mu \mathrm{m}$; b) Ceratium falcatum. $100 \mu \mathrm{m}$; c) Ceratium furca. $100 \mu \mathrm{m}$; d) Ceratium furca var. eugrammum. $100 \mu \mathrm{m}$; e) Ceratium fusus. $100 \mu \mathrm{m}$; f) Ceratium hexacanthum. $100 \mu \mathrm{m}$.

* Ceratium extensum (Gourret) Cleve, 1901.

(Sin.: Ceratium strictum Kofoid, 1907. C. fusus var. stricta Okamura et Nishikawa, 1904. C. biceps Kofoid, 1908).

Encontrado por Herrera \& al. (1955) en ambos meses. También en nuestras muestras aparece en ambos meses.

Longitud de $1180 \mu \mathrm{m}$, diámetro cingular de $30 \mu \mathrm{m}$. Lám. 4, a.

* Ceratium falcatum (Kofoid) Jörgensen, 1920.

(Sin.: Ceratium pennatum f.falcata Kofoid, 1907. C. pennatum var.falcatum Jörgensen, 1911).

Es citado por Margalef \& al. (1957) en muestras de Noviembre. Presente en nuestras muestras del mismo mes.

Longitud de $285 \mu \mathrm{m}$, anchura en el cíngulo de $20 \mu \mathrm{m}$. Lám. 4, b. 


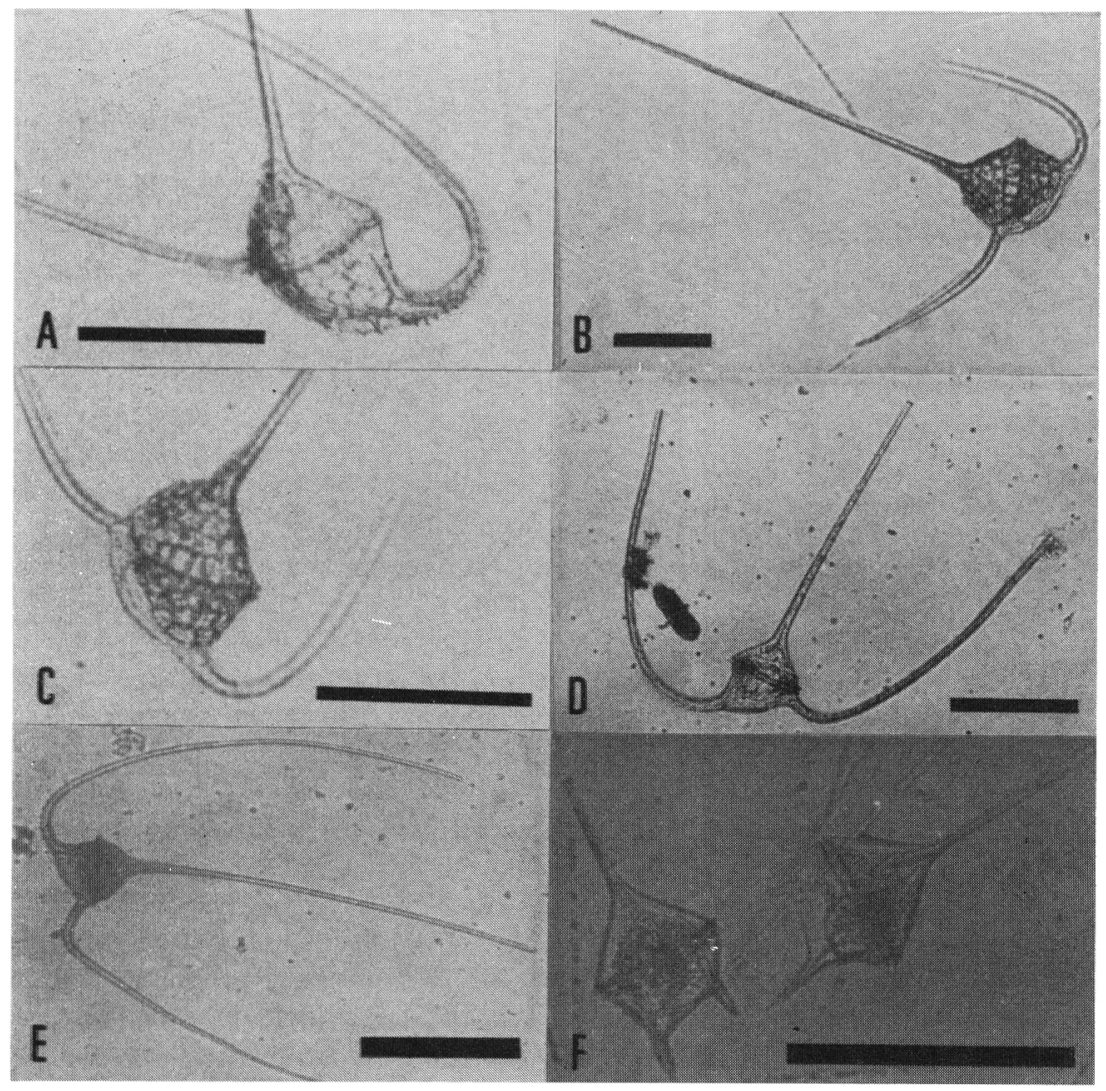

Lámina 5: a) Ceratium hexacanthum. Detalle. $100 \mu \mathrm{m}$; b) Ceratium hexacanthum var. aestuarium. $100 \mu \mathrm{m}$; c) Ceratium hexacanthum var. aestuarium. Detalle. $100 \mu \mathrm{m}$; d) Ceratium horridum. $100 \mu \mathrm{m}$; e) Ceratium macroceros. $100 \mu \mathrm{m}$; f) Ceratium pentagonum var. tenerum. $100 \mu \mathrm{m}$.

* Ceratium furca (Ehrenberg) Clap. et Lachm., 1859.

(Sin.: Peridinium furca Ehr., 1833. P. eugrammum Ehr., 1859. Ceratium biceps Claparède et Lachmann, 1859. Biceratium furca Vanhöffen, 1897).

Hallado por Herrera \& al. (1955) en Noviembre. Lo encontramos en nuestras muestras del mismo mes.

Longitud de $225 \mu \mathrm{m}$, ancho cingular de $32 \mu \mathrm{m}$, cuernos antiapicales de 35 y $75 \mu \mathrm{m}$. Lám. 4, c.

* Ceratium furca (Ehrenberg) Clap. et Lachm., 1859 var. eugrammum (Ehrenberg0 Jörgensen, 1911.

(Sin.: Peridinium eugrammum Ehr., 1859. Ceratium furca var. medium Gourret, 1883. C. furca var. pouchetti Lemmermann, 1900. C. furca var. tertium Gourret, 1883). 


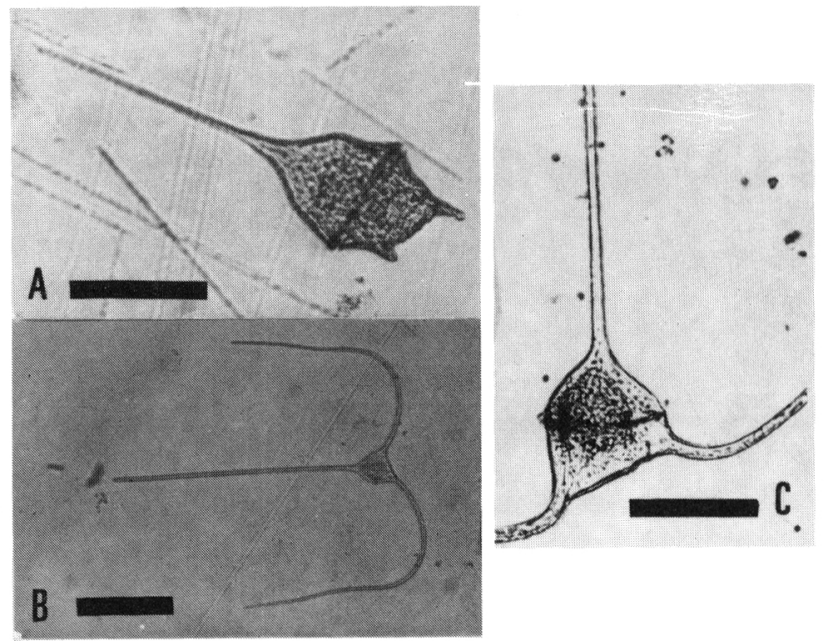

Lámina 6: a) Ceratium pentagonum var. turgidum. $100 \mu \mathrm{m}$; b) Ceratium trichoceros. $200 \mu \mathrm{m}$; c) Ceratium trichoceros. Detalle. $50 \mu \mathrm{m}$.

Lo citan Herrera \& al. (1955) en ambos meses. Presente en nuestras muestras de Noviembre.

Longitud de $210 \mu \mathrm{m}$, anchura en el cíngulo de $45 \mu \mathrm{m}$, cuernos de 35 y $90 \mu \mathrm{m}$. Lám. 4, d. * Ceratium fusus (Ehrenberg) Dujardin, 1841.

(Sin.: Peridiniumfusus Ehr., 1833.P. seta Ehr., 1859. Amphiceratiumfusus Vanhöffen, 1899. Ceratium pellucidum Gourret, 1883. C. berghii Gourret, 1883).

Encontrado por Herrera \& al. (1955) en Noviembre. Aparece en nuestras muestras de ambos meses.

Longitud de $410 \mu \mathrm{m}$, diámetro cingular de $25 \mu \mathrm{m}$. Lám. 4, e.

* Ceratium hexacanhum Gourret, 1883.

(Sin.: Ceratium reticulatum (Pouchet) Cleve, 1903. C. tripos var. reticulata Pouchet, 1883. C. tr. var. inaequale Gourret, 1883).

Citado por Herrera et al. (1955) en Noviembre. Presente en nuestras muestras del mismo mes.

Longitud de $390 \mu \mathrm{m}$, pico de $310 \mu \mathrm{m}$, cíngulo con $70 \mu \mathrm{m}$, cuernos de 300 y $315 \mu \mathrm{m}$, Lám. 4, f y 5 , a.

* Ceratium hexacanthum Gourret, 1883 var. aestuarium (Schröder) Jörgensen, 1920.

Esta variedad de $C$. hexacanthum no aparece citada como tal en los inventarios de los trabajos consultados, no obstante puede haber sido encontrada en ellos y determinada con el nombre de la especie. Presente en nuestras muestras de Noviembre.

Longitud de $510 \mu \mathrm{m}$, pico de $360 \mu \mathrm{m}$, cíngulo con $65 \mu \mathrm{m}$, cuernos de 140 y $160 \mu \mathrm{m}$. Lám. 5 , b y 5 , c.

* Ceratium horridum (Cleve) Gran, 1902.

(Sin.: Ceratium intermedium (Jörg.) Jörg., 1905, C. tripos var. macroceros f. intermedia Jörg., 1899. C. batavum Paulsen, 1908, C. tripos var. horrida Cleve, 1896).

Aunque en los inventarios no aparece con este nombre, sí podría hacerlo como C. tripos, 
citado por Herrera \& al. (1955) durante ambos meses.

Long. de $260 \mu \mathrm{m}$, pico de $200 \mu \mathrm{m}$, cíngulo de $40 \mu \mathrm{m}$, cuernos de 250 y $260 \mu \mathrm{m}$. Lám. 5,d.

* Ceratium macroceros (Ehrenberg) Cleve, 1900.

(Sin.: Peridinium macroceros Ehr., 1840. C. tripos var. macroceros Clap. et Lach., 1859. C. tripos var. scotica Schütt, 1892. C. gallicum Kofoid, 1907. C. macroceros subsp. gallicum (Kofoid) Jörg., 1911).

Citado por Herrera \& al. (1955) en el mes de Octubre. Aparece en nuestras muestras del mismo mes.

Longitud de $315 \mu \mathrm{m}$, pico de $250 \mu \mathrm{m}$, cíngulo con $35 \mu \mathrm{m}$, cuernos de 230 y $320 \mu \mathrm{m}$. Lám. 5, e.

* Ceratium pentagonum Gourret, 1883 var. tenerum Jörgensen, 1920.

(Sin.: Ceratium furca var.pentagonum Lemmermann, 1900. C. furca var. baltica Schütt, 1895. C. lineatum var. longiseta Ostenfeld et Schmidt, 1901. C. lineatum var. robusta Cleve, 1900. C. candelabroides Schimper et Vanhöffen, 1904).

Es la variedad más común de $C$. pentagonum, por lo cual es de suponer que sea la encontrada por Herrera \& al. (1955) durante el mes de Octubre. En nuestras muestras aparece en Noviembre.

Longitud de $140 \mu \mathrm{m}$, pico de $75 \mu \mathrm{m}$, diámetro cingular de $55 \mu \mathrm{m}$. Lám. 5, f.

* Ceratium pentagonum Gourret, 1883 var. turgidum (Jörg) Jörgensen, 1911.

Aunque no se cita tal variedad en los inventarios de los trabajos consultados, puede haberse incluido con el nombre de la especie. Ha sido encontrada durante el mes de Noviembre.

Longitud de $330 \mu \mathrm{m}$, pico de $205 \mu \mathrm{m}$, cíngulo con $70 \mu \mathrm{m}$, cuernos antiapicales de unas $30 \mu \mathrm{m}$. Lám. 6, a.

* Ceratium trichoceros (Ehrenberg) Kofoid, 1908.

(Sin.: Peridinium trichoceros Ehr., 1859.Ceratiumflagelliferum Cleve, 1900.C.tripos flagelliferum Karsten, 1906).

Hallada por Herrera \& al. (1955) durante ambos meses. Aparece en nuestras muestras en ambos meses, principalmente en Octubre.

Longitud de $525 \mu \mathrm{m}$, pico de $475 \mu \mathrm{m}$, cíngulo con $50 \mu \mathrm{m}$, cuernos de $550 \mu \mathrm{m}$. Lám. 6 , b y 6 , c.

\section{AGRADECIMIENTOS}

Este trabajo no habría podido realizarse sin la participación desinteresada de las siguientes personas: Dr. M. Guara Requena (Departamento de Biología Vegetal. Unidad de Fitografía), por su ayuda en la corrección del manuscrito y su apoyo en todo momento. F. Tirado, de la empresa Consulmar, quien puso a nuestra disposición la embarcación para nuestros muestreos. Y F.J. Iborra, por su ayuda en el mecanografiado del original.

\section{BIBLIOGRAFÍA}

DODGE, J.D. -1985- Atlas of Dinoflagellates. Farrand Press, London.

HERRERA, J., F. MUÑOZ \& R. MARGALEF -1955- Fitoplancton de las costas de Castellón durante el año 1953. Investigación Pesquera, 1:17-99. Barcelona. 
HERRERA, J. \& R. MARGALEF -1957- Fitoplancton de las costas de Castellón, de julio de 1956 a junio de 1957. Investigación Pesquera, 10:17-44. Barcelona.

HERRERA, J. \& R. MARGALEF - 1961- Hidrografía y fitoplancton de las costas de Castellón, de julio de 1959 a junio de 1960. Investigación Pesquera, 20:17-63. Barcelona.

HERRERA, J. \& R. MARGALEF -1963- Hidrografía y fitoplancton de la costa comprendida entre Castellón y la desembocadura del Ebro, de julio de 1960 a junio de 1961. Investigación Pesquera, 24:33-112. Barcelona.

LOPEZ, J. -1955- Variación alométrica en Ceratium tripos. Investigación Pesquera, 2:131159. Barcelona.

LOPEZ, J. -1966- Variación y regulación de la forma en el género Ceratium. Investigación Pesquera, 30:325-427. Barcelona.

MARGALEF, R., M. DURAN \& F. SAINZ - 1955- El fitoplancton de la Ría de Vigo de enero de 1953 a marzo de 1954. Investigación Pesquera, 2:85-129. Barcelona.

MARGALEF, R., F. MUÑOZ \& J. HERRERA -1957- Fitoplancton de las costas de Castellón de enero de 1955 a junio de 1956. Investigación Pesquera, 7:3-31. Barcelona.

MARGALEF, R., J. HERRERA \& E. ARIAS -1959- Hidrografía y fitoplancton de las costas de Castellón, de julio de 1957 a junio de 1958. Investigación Pesquera, 15:3-38. Barcelona.

MARGALEF, R. \& J. HERRERA -1963- Hidrografía y fitoplancton de las costas de Castellón, de julio de 1959 a junio de 1960. Investigación Pesquera, 22:49-109. Barcelona.

MARGALEF, R. \& J. HERRERA -1964- Hidrografía y fitoplancton de la costa comprendida entre Castellón y la desembocadura del Ebro, de julio de 1961 a julio de 1962. Investigación Pesquera, 26:49-90. Barcelona.

MUÑOZ, F., J. HERRERA \& R. MARGALEF -1956- Fitoplancton de las costas de Castellón durante el año 1954. Investigación Pesquera, 3:75-90. Barcelona.

MUÑOZ, F. \& J.M. SAN FELIU -1972- Hidrografía y fitoplancton de las costas de Castellón, de julio de 1968 a junio de 1969. Investigación Pesquera, 36(2):365-392. Barcelona.

RABENHORST, L. -1933/1937- Kryptogamen-Flora: Flagellatae; Dinoflagellatae. Teil, 1 (1933) - Teil, 2 (1937). Akademische Verlagsgesellschaft M.B.H. Leipzig.

RAMPI, L. \& M. BERNHARD -1980- Chiave per la determinazione delle peridinee pelagiche mediterranee. Comitato Nazionale Energia Nucleare. Roma.

SAN FELIU, J.M. \& F. MUÑOZ - 1967- Hidrografía y fitoplancton de las costas de Castellón, de mayo de 1965 a julio de 1966. Investigación Pesquera, 31(3):419-461. Barcelona.

SAN FELIU, J.M. \& F. MUÑOZ - 1970- Hidrografía y fitoplancton de las costas de Castellón, de julio de 1967 a junio de 1968. Investigación Pesquera, 34(2):417-449. Barcelona.

SAN FELIU, J.M. \& J. MUÑOZ - 1975- Hidrografía y fitoplancton de las costas de Castellón, de septiembre de 1969 a enero de 1971. Investigación Pesquera, 39(1):1-35. Barcelona. TREGOUBOFF, G. -1957- Manuel de Planctonologie méditerranéenne. C.M.R.S. Paris.

UNESCO -1981- Phytoplankton manual. Edited by A. Sournia. Muséum National d'Histoire Naturelle. Paris.

(Aceptado para su publicación en Junio de 1.990 )

Dirección del autor: Departamento de Biología Vegetal. Unidad de Fitografía. Facultad de Ciencias Biológicas. Universidad de Valencia. C/ Dr. Moliner, 50. 46100 Burjasot (Valencia). 